\title{
LA DESVALORIZACIÓN DEL TRABAJO AGRÍCOLA
}

\author{
Matías Berger ${ }^{1}$ \\ Elena Mingo ${ }^{2}$
}

\section{Introducción}

En este artículo nos proponemos analizar la 'desvalorización' del trabajo agrícola a partir del discurso de trabajadores, empleadores y funcionarios en el Valle de Uco, provincia de Mendoza (Argentina). Con ‘desvalorización’ nos referimos a la enunciación de nociones sobre el trabajo agrícola que lo califican como un trabajo que se aprende con facilidad, poco calificado, que no requiere demasiado tiempo de capacitación y aprendizaje, incorporado en un proceso de trabajo con tareas poco diferenciadas y realizado por los segmentos de población con menos instrucción formal, mayores dificultades para encontrar ocupación, menores ingresos y potenciales perceptores de las políticas públicas de asistencia social.

El objetivo de este artículo es analizar la forma en que esas nociones se manifiestan en los relatos de los agentes y nos permiten comprender la desvalorización como un proceso social con diferentes aristas. Los materiales para la elaboración del artículo son producto de un extenso trabajo de campo en la zona realizado entre los años 2005 y 2010 consistente en entrevistas con trabajadores, empleadores y funcionarios que han permitido dar continuidad a las reflexiones sobre el trabajo agrícola.

La provincia de Mendoza se destaca por la vitalidad y diversificación de su sector agropecuario vinculado tanto al mercado interno como al externo, fuerte demandante de mano de obra, eje de innovaciones tecnológicas, diverso en la gama de agentes económicos que lo componen, articulado con etapas de transformación industrial, eje y motor de procesos de acumulación ensamblados con la realidad provincial y sustancia de sectores económicos entrelazados en la vida social y política a través de múltiples instituciones. Este elemento imprime una identidad particular a la provincia diferenciando su realidad social

\footnotetext{
1 Centro de Estudios e Investigaciones Laborales/ Consejo Nacional de Investigaciones Cientificas y Tecnicas, Argentina.

${ }^{2}$ Centro de Estudios e Investigaciones Laborales/ Consejo Nacional de Investigaciones Cientificas y Tecnicas, Argentina.
} 
no sólo en su configuración económica, sino también por la particular constitución del mercado laboral.

Si la diversificación productiva y la articulación con la agroindustria nos permiten observar la potencialidad económica de las localidades mendocinas, la situación del empleo nos muestra el rostro atávico de la vida económica, fundamentalmente de las actividades primarias. El sector primario se destaca la proporción de trabajadores no registrados mientras que si bien el sector agroindustrial exhibe mejores indicadores de trabajo registrado no está exento de formas de contratación que privan a los trabajadores de los derechos propios de una relación salarial. Es el caso de las empresas de intermediación y/o contratación eventual que si bien permiten acceder a una relación laboral en forma registrada desdibujan relaciones laborales más estables de lo que aparecen el mediar las mencionadas empresas. A esta realidad laboral es menester agregar la marcada estacionalidad de las posibilidades laborales que afecta los ingresos y aportes de los trabajadores. De más está decir que la precariedad e informalidad de las relaciones laborales afecta las posibilidades de acceso a protección y seguridad social de los trabajadores, fundamentalmente en lo que respecta a las posibilidades de jubilarse, a la antigüedad, al acceso a servicios de salud (amen de la prolongación de las coberturas para el caso de los trabajadores transitorios) y a los ingresos, excepción hecha de los minoritarios puestos de trabajo como permanentes en las fincas, a veces también pasados por el filo de la precarización.

El principal garante del acceso a protección y seguridad social, en todas las localidades, es el Estado, con diferentes aportes en sus distintos niveles de gobierno. En primer lugar, a través de los servicios de salud y educación básicos. En segundo lugar, a través de las políticas públicas de asistencia básicamente expresadas en el otorgamiento de la Asignación Universal por Hijo y en las pensiones y jubilaciones no contributivas. Difícilmente un sector importante de la población de todas las localidades percibiría estos beneficios sociales si ello dependiera de la posibilidad de incorporarse o haber estado incorporado en el pasado en relaciones salariales estables. Sea por causa del desempleo o por causa de la precariedad o informalidad laboral.

En el sector agropecuario y en la agroindustria, el empleo precario ha sido una tendencia histórica, reforzada en los '90 por el sustento de las políticas públicas y la falta de 
fiscalización estatal y de allí la importancia de las ya mencionadas políticas del sector público.

En primer lugar, el artículo describe las características productivas y de los mercados de trabajo en la provincia de Mendoza en clave histórica. Luego pasamos al análisis de los datos de campo generados. Así, analizamos las características y formas en que se desarrolla el aprendizaje del trabajo agrícola a partir de los relatos de trabajadoras/es entrevistados. Luego analizamos la falta de reconocimiento de los saberes necesarios para desempeñarse en trabajos agrícolas, el ocultamiento de esos saberes detrás de otras características y la adscripción a puestos de trabajo en relación a las cualidades personales y a patrones de género y clase. Por último, analizamos la forma en que se naturalizan los saberes y así son desconocidos en las remuneraciones y en las trayectorias ocupacionales de las/os trabajadoras/os.

\section{La configuración productiva en la provincia de Mendoza}

La provincia de Mendoza se encuentra ubicada en el centro-oeste del territorio argentino, distante a 1100 kilómetros de la Ciudad de Buenos Aires. Limita al norte con la provincia de San Juan, al este con la provincia de San Luis, al sudeste con La Pampa y al sudoeste con Neuquén. Al oeste limita con la República de Chile, límite internacional establecido por la Cordillera de los Andes. La ubicación geográfica constituye un aspecto fundamental que explica el modelo de desarrollo agroindustrial de la provincia, no solo por sus aptitudes ecológicas sino también por las condiciones políticas que permitieron establecer las bases de acumulación en el desarrollo del comercio con Chile.

A nivel de sus aptitudes agroecológicas el territorio provincial presenta una geografía desértica que al aprovechar, bastante tempranamente en la historia del país, el agua proveniente de los ríos que descienden de la Cordillera de los Andes, permitió la formación de oasis de riego donde se desarrollaron las agroindustrias que hasta hoy configuran sus principales recursos productivos.

El proceso de acumulación de capitales, que luego permitió el desarrollo del modelo agroindustrial, estuvo basado en el comercio de ganado con Chile aprovechando la ventaja que significaba la cercanía con este país. El modelo productivo basado en la ganadería destinada al mercado chileno y en la producción de cereales dirigida al mercado local 
aportó, además, al desarrollo del sector comercial y de servicios vinculado a estas actividades.

Por otro lado, la conformación de una elite agraria provincial forjada alrededor de la ganadería de engorde es un factor de vital importancia para el desarrollo de la actividad vitivinícola posterior. El engorde de ganado, más rentable que la cría, permitió una mayor acumulación de capitales y también influyó en las características comerciales imprimiendo mayor dinamismo a estos sectores productivos. El dinamismo atribuido a las elites ganaderas de la provincia, sumado al desarrollo de otras actividades conexas como el control de la actividad forrajera, la producción de cereales y harinas y el control del comercio y el transporte les permitieron acumular importantes sumas de capitales sobre las que recayó parte del financiamiento de la reconversión hacia la vitivinicultura a partir de 1870 ya que a fines de la década de 1860 la actividad ganadera-comercial entra en crisis (Gómez, 2001).

Una multiplicidad de factores hace que la actividad económica de la provincia se oriente a la vitivinicultura como respuesta a la crisis de la ganadería comercial; entre ellos se puede enumerar la tradición previa que tenía la provincia en el cultivo de vid, que a finales del siglo XVIII se plasma en la incipiente industria vitivinícola. Por su parte, el ferrocarril, que llega a la provincia hacia 1885, facilita el proceso de modernización de la producción por la importación de maquinarias a la vez que permite el acceso de los vinos mendocinos a los grandes centros urbanos, paralelo al accionar de una clase dirigente que, apoyada por las elites locales, plantea un rediseño de la estrategia productiva estimulando la implantación del cultivo de vid a través de instrumentos de política fiscal. A su vez, en función de dar solución a la problemática de escasez de mano de obra, el poder ejecutivo provincial diseñó políticas dirigidas a fomentar la inmigración europea a la región, especialmente dirigidas a atraer personas que tuvieran conocimientos tanto del cultivo de vid como de la producción de vinos (Cerdá, 2005).

En resumen, hacia el final del siglo XIX múltiples causas influyen en la conformación del modelo productivo provincial. Por un lado, el afianzamiento del estado nacional a partir del cual se organiza el mercado interno y, por el otro, la consolidación de una división del trabajo regional a partir de la cual la provincia se incorpora al modelo agroexportador predominante en el territorio nacional (Cerdá, 2005). Así, la provincia de 
Mendoza se especializó en la producción de uvas destinadas a la fabricación de vinos que se vio favorecida por un aumento importante en el consumo de vinos producto de la inmigración española e italiana que se establece en el territorio nacional (Richard-Jorba, 2005).

No obstante, el modelo vitivinícola basado en la producción de grandes volúmenes para el consumo en el mercado interno atravesó sucesivas crisis durante todo el siglo XX puesto que el esquema se encontró atado a la fuerte dependencia de las oscilaciones del consumo interno y a los precios de la uva y el vino (Mateu, 2007).

En relación con esto último, si bien se verifica en la historia provincial una centralidad de la producción de uvas y vinos, es también importante la presencia de cultivos frutales para consumo en fresco pero también con destino a procesamientos agroindustriales. En este sentido, la diversificación productiva en la provincia ha sido, a lo largo de su historia reciente, una de las estrategias implementadas desde la dirigencia local para disminuir los efectos de las múltiples crisis que atravesó la vitivinicultura a lo largo del siglo $\mathrm{XX}$.

En el territorio del Valle de Uco se observan los procesos mencionados; a la vez que ha conformado históricamente una zona de producción tanto vitivinícola como frutícola, también ha sido protagonista de los diversos procesos de transformación. La confluencia de cambios y transformaciones de orden productivo, institucional y económico impulsaron, a partir de la década de 1960, una importante diversificación de la estructura agraria que se expresó en el crecimiento de la superficie cultivada de frutales y hortalizas (Bocco, Martín y Pannuzio, 1999).

\section{Conformación del mercado de trabajo}

Como se mencionó más arriba, el proceso atravesado por la provincia en la orientación y definición de su modelo productivo se destaca por la conformación temprana de una burguesía provincial en combinación con una clase dirigente que, como es lógico, pertenecía también a los sectores productivos. Ello permitió reorientar las políticas vinculadas a fortalecer el proceso de transición del modelo de producción, como por ejemplo la incorporación de tecnología vinculada al riego y también el fomento a inmigración europea para atraer mano de obra calificada en la producción de uvas y vinos. 
Richard-Jorba (2001) señala que a pesar de que persistieron algunas características de la estructura social sobre la que se asentaba el modelo anterior que complejizaron la consolidación de un 'mercado de trabajo libre’, se destaca que el modelo vitivinícola abrió paso al surgimiento de una clase de pequeños y medianos propietarios vinculada a la inmigración europea que acrecentó los sectores medios locales. Esto se reflejó, por ejemplo, en la nueva conformación de actores sociales que derivo del modelo de desarrollo capitalista en la provincia. La nueva estructura social, conformada por viñateros, productores agroindustriales, industriales bodegueros, bodegueros integrados, comerciantes extrarregionales y la figura del contratista de viña ${ }^{3}$, estaban también vinculadas a través de relaciones asimétricas.

Los cambios económicos y políticos tuvieron repercusión en la sociedad mendocina que se convertiría en una sociedad más compleja y heterogénea hacia finales del siglo XIX. Estos cambios están vinculados a la influencia que tuvo la inmigración en la estructura demográfica, el desarrollo urbano y la consolidación de estratos medios en conjunto con la aparición de los sectores obreros (Richard-Jorba, 2001).

En lo que refiere a la conformación del mercado de trabajo vinculado a la producción vitivinícola, el autor destaca la influencia que tuvieron las estructuras sociales características del siglo XIX. En este sentido, menciona que hacia la mitad del siglo la clase trabajadora permanecía prácticamente excluida de la sociedad con límites estrictos reflejados, por un lado, en los ingresos percibidos con los que apenas alcanzaban la reproducción y, por otro, por un marco de regulación y control a través del cual se ejercía una fuerte coacción sobre los trabajadores.

Hacia fines del siglo XIX, las elites que controlaban la economía y el estado ejercían un fuerte paternalismo sobre las clases no propietarias. En este contexto, señala Richard- Jorba (2001), el discurso hegemónico sobre el que se fundamentaba el control social interpretaba al trabajo como una obligación y no como un derecho. Sobre estas interpretaciones se fundamentaba la explotación de aquellos que poseían solo su fuerza de trabajo.

\footnotetext{
${ }^{3}$ El contratista de viña, figura que perdura hasta hoy pero en pocos casos, tenía a su cargo una explotación en la que trabajaba con su familia y recibía un porcentaje de la venta de la producción. Los contratistas de viña no eran propietarios de la tierra, aunque en algunos casos esta posición permitió procesos de acumulación que vehiculizaron la compra de tierras, mientras que en otros se produjo un proceso de "proletarización" de los contratistas y sus familias.
} 
El control de la mano de obra se ejercía a través de un marco jurídico que reglamentaba la duración de los contratos fundamentados en una supuesta falta de "apego al trabajo" por parte de los trabajadores. De esta forma, el estado, a través de la policía, controlaba el mercado de trabajo restringiendo la movilidad geográfica de los trabajadores. El instrumento de control utilizado era la llamada "papeleta de conchabo" ya que sin este documento los trabajadores no podían ser contratados; además, el hecho de no portarlo implicaba la posibilidad de ser detenido bajo la acusación de vagancia y forzado a trabajar durante un período de tiempo para el estado. Por otra parte, constituye un dato interesante el discurso con el que las clases propietarias se referían a las/os trabajadoras/es. En este sentido, el autor cita una solicitada publicada en enero de 1864 donde se conmina a las autoridades locales a ejercer las disposiciones de control sobre los trabajadores: “[...] se encontrarán ranchos de la clase pobre, llenos de muchachos de ambos sexos poco menos que desnudos, sin ninguna ocupación y aprendiendo toda clase vicios. ¿No sería mejor que se obligase a esos padres idiotas a que colocasen a sus hijos en casas respetables donde serían útiles, al mismo tiempo que se moralizarían?” (Richard-Jorba, 2001: 5). De esta forma se expresaba que la supuesta inmoralidad de las clases trabajadoras era la causante de la falta de mano de obra. Aunque escrito hace casi 150 años, este imaginario sobre las causas de la falta de mano de obra prevalece en la zona aunque sostenido en otros argumentos más acordes con la época que aún continúan poniendo en cuestión la moral de las/os trabajadoras/es. Ejemplo de esto es la relación causal que establecen los agentes vinculados a la producción entre la distribución de planes de asistencia social y la falta de mano de obra estacional.

Aunque los mecanismos coercitivos buscaban limitar la movilidad de los trabajadores durante segunda mitad del XIX no evitaron el desarrollo de un mercado de trabajo libre y unificado. En este sentido, se destaca la importancia que tuvo la participación de la mano de obra de origen europeo no solamente por el aumento de la oferta de mano de obra sino por las resistencias que esta presentaba a los mecanismos de coacción (Richard-Jorba, 2001).

La escasez de mano de obra se convirtió en un problema para el desarrollo del cultivo de la vid en la provincia; por ello los empresarios vinculados al sector, que a su vez 
tenían fuertes vínculos con los funcionarios gubernamentales, solicitaron a estos el desarrollo de campañas que promovieran la inmigración (Cerdá, 2006).

A partir del siglo XX el mercado de trabajo se caracteriza, por un lado, por una demanda de mano de obra permanente muy reducida y, por otro, por una gran cantidad de trabajadoras/es disponibles para realizar tareas temporarias a lo largo del año. El autor concluye que el modelo de desarrollo provincial generó largos períodos de inactividad y una alta inestabilidad en los ingresos de la población (Cerdá, 2005). Ambas circunstancias continúan existiendo aún en la primera década del siglo XXI.

En definitiva, los estudios históricos citados confirman que algunas características observadas en la conformación del mercado de trabajo, vinculado a la vitivinicultura a fines del siglo XIX, guardan correspondencia con la actualidad de estos espacios laborales. Nos referimos a las condiciones precarias de trabajo y a la inestabilidad laboral como también a la alta demanda de mano de obra temporaria frente a una escasa cantidad de puestos de trabajo permanentes. En cuanto al control de la mano de obra, si bien han desaparecido aquellos instrumentos legales represivos destinados a intervenir en la movilidad territorial de las/os trabajadoras/es, permanece el control social a través de los límites impuestos por los bajos salarios percibidos que en la actualidad mínimamente alcanzan para la reproducción de los hogares de los trabajadores. Otra forma de control social en la que encontramos líneas de continuidad está vinculada a los discursos sobre la clase trabajadora. A través de una diversidad de aseveraciones tanto desde los sectores productivos como de otros agentes sociales (maestras/os, comerciantes, agentes de salud, fuerzas de seguridad, etc.) se continúa calificando a las/os trabajadoras/as agrícolas como "vagos" adjudicando la imposibilidad de una movilidad social ascendente al "desapego por el trabajo" y no a los bajos salarios percibidos, la discontinuidad laboral y la desprotección legal ejercida sobre ellas/os.

\section{El aprendizaje del trabajo}

El primer aspecto que analizaremos se refiere a la forma en que los trabajadores aprenden a trabajar, teniendo en cuenta cómo es considerado ese aprendizaje por parte de trabajadores y empleadores y las disposiciones que ese aprendizaje genera, en particular las 
referidas a la incorporación al mundo del trabajo y a las posibilidades de continuar con trayectorias educativas.

Cuando la entrevistamos en diciembre de 2006, Olga tenía cuarenta años, un hijo y vivía en la casa de sus padres en una pequeña localidad llamada Eugenio Bustos. Ella relata que empezó a trabajar cuando tenía diez años en las cosechas de tomate, uva y nuez, en la atada de viña y en otras tareas en la fruticultura. Olga aprendió a trabajar la tierra junto a su familia, primero ayudando en la chacra familiar, en la que su padre era contratista, y luego haciendo otro tanto en trabajos eventuales en otras fincas.

Su aprendizaje del trabajo sucedió en el grupo familiar y su búsqueda de trabajo fuera de la chacra lo atribuye a la falta de recursos en su hogar. Olga señala que el trabajo en la tierra es cansador y que resulta muy difícil trabajar y continuar estudiando. La mención al "estudio" surge como parte de una dimensión sobre la que se indagaba en la entrevistas, la trayectoria educativa. Mientras que algunos entrevistados asociaban la educación con las probabilidades de conseguir un 'buen trabajo' o un 'mejor trabajo', señalándola como un factor de acumulación, otros entendían que la 'escuela' competía con sus posibilidades laborales restándoles tiempo e ingresos. Además, la indagación sobre las trayectorias educativas permite comprender la necesidad e importancia de la ubicación geográfica y de las posibilidades de acceso a servicios. Durante la entrevista ella habló sobre el trabajo en la tierra y lo que ella desea para su hijo:

Estudiaba de noche pero me canse porque el trabajo de la tierra cansaba mucho, entonces con mi hijo voy a luchar para que estudie, para que tenga su empleo bueno y trabaje porque es muy duro.

Este primer testimonio da cuenta de algo que aparecerá en otros de manera reiterada: una iniciación laboral durante la niñez. Pero además, en el testimonio transcripto no sólo se plantea la dificultad para trabajar y estudiar dado lo agotador de los trabajos agrícolas sino que el 'estudio' es asociado a la posibilidad de realizar trabajos fuera de la agricultura, de tener un 'buen empleo'.

Otro trabajador entrevistado, Alberto, de 41 años, cuenta que empezó a trabajar por su cuenta a los catorce años aproximadamente. A esa edad dejó de ir a la escuela sin haber llegado a terminar la primaria: 
R: ...si, empecé trabajando con mi viejo, un decir, al lado de mi viejo y después cuando era... ya más adelante, me fui a trabajar solo...

P: ¿Y en que trabajaste, en que empezaste con tu papá... qué hacías?

R:...colectando tomate, plantando, cuando era el orégano, antes plantaba mucha menta también...

Menciona que uno de los motivos por los que dejó la escuela es que no tenía ropa para ir y dice que 'antes no era igual que ahora, que se está un poquito mejor'. Alberto vive con su esposa, Nidia, con quien tienen tres hijos. Nidia trabaja eventualmente en la atada, durante el invierno, y en la cosecha de vid, en verano. También trabajan dos de sus hijos. Uno de ellos, de 19 años, terminó la primaria y se dedica a trabajar en una finca vitivinícola. El otro hijo acompaña a la madre a su trabajo. Le preguntamos a Alberto por qué los hijos empezaron a atrabajar:

$\mathrm{R}:$...no ellos nomás se fueron, saben que tienen que trabajar y ganar plata para poder vivir...

Sostiene Alberto que sus hijos "saben que tienen que trabajar". Si bien uno de los hijos de Alberto logró terminar la primaria, a diferencia de su padre, no pudo continuar estudiando porque ello implicaba ir diariamente al pueblo, distante varios kilómetros, ya que en el lugar en el que residen no hay escuela secundaria. Alberto, Nidia y sus hijos viven en el paraje 'El Cepillo', un barrio rural en el departamento de San Carlos.

En el transcurso de las entrevistas preguntábamos por la iniciación laboral de los padres de los entrevistados y de los hijos, además de la propia del entrevistado, con el objetivo de conocer la mediación de los vínculos familiares en las inserciones laborales y los distintos aprendizajes que ocurrían en ese proceso.

Durante la entrevista, Ricardo, de 45 años, nos contó la forma en que su hijo empezó a trabajar por su cuenta a los 15 años:

P: ¿y en el principio vos le enseñaste el oficio, vos le enseñaste a trabajar, empezaba a salir con ustedes así?

R: sí, nosotros, cuando iba con nosotros le enseñábamos [se escucha de fondo a la madre decir que cuando el hijo tenía 5 años ella lo tenía que llevar al trabajo], él aprendió ahí igual. En ese entonces él lo hacía porque quería nomás; entonces él iba ahí y aprendía, a la par de nosotros iba aprendiendo; después, ya cuando tuvo quince años, ya salió con el Rubén [su primo], si no sabía le enseñaba él ahí viste, a ralear, a podar, a hacer... todo trabajo que él no lo sabía aprendió ahí con él. 
Ricardo vive con su esposa y su hijo en la zona de Cordón del Plata, un barrio rural a unos 30 kilómetros de la ciudad de Tupungato. Trabaja al tanto en forma eventual en las cosechas, en raleos y en la poda de vid. En este testimonio se refleja que el aprendizaje del trabajo se realiza a partir de la transmisión de los saberes por parte de con parientes y/o amigos que transmiten los conocimientos de las tareas sin que medie ninguna instancia de aprendizaje formal ni se genere certificación alguna de los saberes adquiridos.

Mario, un joven trabajador entrevistado (19 años), narra que empezó a trabajar con sus padres cosechando uvas, tomates, manzanas y cereza:

R: Ahí, acostumbrándote para poder empezar cuando seas más grande. Y después agarramos $(. .$.$) con mi hermano y salimos a trabajar, él por su parte, yo por la mía y mi$ hermano por ahí.

Mario terminó la escuela primaria y luego, a pesar de tener intenciones de continuar estudiando, se dedicó exclusivamente a trabajar:

P: ¿Y con el estudio? Pudiste hasta que...

R: Salí de la escuela primaria acá y me fui a laburar.

$\mathrm{P}:$ ¿Y la secundaria no pudiste hacer?

R: No, pasa que somos tres y tenes que trabajar... y los tres, hay que trabajar, no te podes parar porque tu viejo gana pero vos tenes que ganar también.

Actualmente, Mario está contratado a través de una empresa que brinda servicios eventuales y trabaja unos cinco meses al año en forma continua. En el momento en que lo entrevistamos, esa empresa contrataba cuadrillas de trabajadores que se desempeñaban en tareas temporarias en una gran empresa vitivinícola integrada de la zona que tercerizaba las contrataciones a través de la empresa que contrataba a la persona entrevistada. Mario fue uno de los pocos trabajadores que comentó haber recibido capacitaciones para realizar los trabajos pero dichas capacitaciones tampoco no eran certificadas por ninguna institución.

Otro joven trabajador, Maxi, de 18 años, relató durante la entrevista que le realizamos que trabajaba desde los diez años. En diciembre de 2006, momento de la entrevista, Maxi trabajaba de tractorista en una chacra desde hace dos años. Además, trabajaba en un matadero de ganado vacuno. Relató que empezó a trabajar con su padre y luego decidió dejar la escuela y dedicarse a trabajar. Le preguntamos que lo llevó a tomar esa decisión:

R: ...y porque, hay muchas cosas, porque por ahí quería salir y no tenía plata y no me gustaba salir sin plata, y entonces me fui a trabajar, y abandoné la escuela, trabajaba muy 
poco si llegaba de la escuela y salía a trabajar pero tampoco podía, tuve que abandonar la escuela...

Con su padre, Maxi trabajaba en distintos lugares haciendo trabajos en forma eventual: "R: ... sí, era una semana, dos semanas, y ahí cambiábamos de lado, éramos así como changarines..."

Como señalamos anteriormente, en el caso de Maxi, la posibilidad de tener su propio ingreso lo llevó a privilegiar la posibilidad de trabajar más tiempo y dejó la escuela. La competencia entre la 'escuela' y el 'trabajo' se presenta a través de una gama de motivos básicamente vinculados a la colaboración en el hogar, las posibilidades de conseguir algo propio, la intención de satisfacer necesidades personales que se incrementan en la adolescencia y se contrastan con las carencias de la infancia y, por último, las dificultades de los grupos familiares para sostener la acumulación en las trayectorias educativas.

María, de 20 años, trabaja en la tierra hace cinco. También empezó a trabajar porque quería comprarse sus propias cosas. Empezó trabajando en una gran empresa de la zona y ahora trabaja en otra. En esa primera empresa trabajaba como temporaria durante tres meses y luego cosechaba en otra finca pequeña, más cerca de su casa. La primera vez fue con unos amigos:

P: ¿Por qué fuiste a trabajar la tierra?

R: Porque no pude seguir la escuela porque en mi casa éramos muchos y mi papá solo, y también tenía que faltar mucho a la escuela. Porque en ese momento mi mamá trabajaba y yo me quedaba con mis hermanos más chicos.

Antonio tiene 58 años, es padre de siete hijos y, en el momento en que lo entrevistamos, vivía con su esposa y dos de sus hijos ya adolescentes. Cuenta que empezó a trabajar ayudando a su padre, que era contratista de viña:

Sí, yo empecé de chiquito, antes se trabajaba con los padres, contrato. La viña se trabajaba siempre y así después en distintos trabajos, cuando terminábamos de trabajar en contrato se trabajaba con otros contratistas. Pero el trabajo es el mismo. Todo vid, siempre la vid trabaje... ...yo a los 9 años ya estaba trabajando...

Antonio comenzó a trabajar ayudando a su padre que era contratista de viña y actualmente es empleado permanente en una finca en la que se hace vid. A pesar de ser permanente y conocer el oficio, Antonio cobraba el salario mínimo y no le pagaban los días 
no trabajados por razones climáticas ni los días que faltaba por razones de salud. Cuenta las tareas que hacían de niños, en general las más livianas: “ ...y lo ponían a hacer regueras, surcos en los primeros claros, lo mas livianito para que fuéramos aprendiendo".

Y por último la forma en que el padre distribuía el dinero cobrado:

\begin{abstract}
...Nosotros trabajábamos así con mi padre: mi padre cuando cobraba a fin de año nos daba una plata a nosotros, para vestirse en el año, tener... y así cuando cobraba mensualidad le daba para que uno saliera al cine, para todas esas cosas vio. Para tener para los gastos de uno vio. Pero no una cantidad vio, enorme, pero le daba siempre...
\end{abstract}

Antonio, casado con Yolanda, comenta que sus hijos estudiaron y que él prefería que estudiaran a que trabajaran la tierra. Sin embargo, además de estudiar también aprendieron a 'trabajar la tierra'. La clasificación de tareas tiene un correlato en la clasificación de trabajos que podemos observar en la comparación que hace Antonio entre el trabajo en la tierra y otros trabajos en general y mediante la cual explica las razones por las que ha propiciado que sus hijos tengan la experiencia de trabajar en la tierra aunque estudien:

...sí, sí, estudian y trabajan ellos. Porque no se les va a caer... para ellos es un bien. A parte como... veo yo si quiere elegir el estudio, lo va a elegir porque el trabajo es pesado, el trabajo de la viña. Entonces va a poner empeño para el estudio.

Al igual que Antonio, sus hijos empezaron a trabajar jóvenes, aunque lo hicieron unos años más tarde que su padre, en la adolescencia. Tanto Antonio como Yolanda han tenido trabajo en forma relativamente continua aunque no siempre en forma permanente ni registrada y han podido lograr que sus hijos tengan la oportunidad de estudiar. Cabe destacar que residen en una pequeña localidad cerca de La Consulta que les permite acceder a servicios educativos. Algo a destacar, que hemos señalado ya más arriba, es que las mejores posibilidades laborales de sus hijos han significado que ellos trabajen fuera del sector agrícola.

Los trabajadores relatan experiencias que revelan una iniciación en el trabajo temprana mediada por el grupo familiar y en cuyo transcurso aprenden tareas, incorporan la experiencia de la rotación por distintos trabajos que constituyen un ciclo laboral y también a clasificar las tareas (trabajos livianos y pesados), lo que también supone la incorporación 
de criterios comparativos con otros trabajos y de la noción de esfuerzo. En el esfuerzo se expresa la importancia del soporte grupal de las trayectorias educativas y laborales y el 'costo' de romper con trayectorias de trabajo en la tierra.

En muchos de estos casos de iniciación temprana, convertirse en un trabajador a cambio de una remuneración no significa iniciarse en una relación salarial con su marco de regulación (flexible o no, pero marco al fin). Contrariamente, en muchos casos implica la socialización en aquellos comportamientos y conductas que permiten acceder a un trabajo y sobre las condiciones y relaciones laborales (inestabilidad, remuneraciones insuficientes y trabajo no registrado).

Dicha socialización se constituye como una serie de experiencias que forman 'para' el trabajo, es decir que constituyen subjetiva y objetivamente al trabajador para desempeñarse en tanto tal en forma concreta en realidades y condiciones particulares que conforman disposiciones en tanto condiciones de posibilidad y horizonte práctico. Por lo tanto, la socialización constituye una experiencia que contiene el aprendizaje de un oficio con el correspondiente conocimiento de tareas y también implica una disposición corporal y mental.

\section{Saberes no reconocidos en la adscripción a los puestos de trabajo y el ritmo del proceso de trabajo}

La pregunta posterior que nos hemos hecho es cómo se interpreta ese aprendizaje del trabajo agrícola que no es formalizado. En primer lugar, ¿es reconocido ese aprendizaje? Y ¿Cómo se valora el trabajo hecho? Luego, hemos tratado de recuperar los testimonios de los trabajadores en los que relatan trabajos y la forma de hacerlos para así comprender los saberes vinculados a esas tareas preguntándonos por el reconocimiento social y formal de ese aprendizaje.

Al referirse a los trabajadores transitorios jóvenes que se contratan en una finca, un encargado de finca entrevistado sostiene lo siguiente:

...les cuesta si no los sacan de más chicos a trabajar, después cuando son grandes les cuesta mucho. ... hay gente que nunca trabajó en estas cosas. Pibes de 17 ó 16 años que nunca han hecho este trabajo y lo que pasa es que son gente, digamos, por ahí de más del centro, que están acostumbrados a la escuela, estudian nomás y cuando les toca salen a trabajar, no están acostumbrados digamos, no se adaptan mucho... 
La caracterización describe espacios de socialización (el centro, simbolizando la ciudad y la escuela) formas en que ella es impulsada (hay que sacarlos de chicos) y, esa forma, contiene una disposición particular que luego hace que no les cueste el trabajo. Allí se reconocen condiciones necesarias para trabajar vinculadas al sacrificio, el esfuerzo pero en menor y escasa medida a destrezas y conocimientos.

En otra parte, el mismo entrevistado afirma que el problema es cuando no saben cosechar, para lo que es muy importante tomar la fruta del tamaño adecuado (saber tamañar). La tarea del encargado es explicar cómo tienen que hacer esa selección, que es muy importante en la primera pasada:

...si no te agarran bien, como vos le enseñás, te cuesta mucho..., cuando haces la primera pasada tenes que hacer todo lo grande. ...por ahí alguien que no sabe, viene, tironea, te rompe la planta y así te va rompiendo la cosecha que viene...

En relación a la edad del aprendizaje y a la influencia de la escolarización, Angie, una joven trabajadora de 18 años, se refiere a la experiencia que han tenido en su familia con sus hermanos. También señala diferencias entre quienes empezaron a trabajar de pequeños y aquellos que no lo hicieron y salen a acompañar en el trabajo siendo más grandes:

El año pasado para el tiempo de cosecha me lo llevé a mi hermano que nunca había salido a trabajar. Lo puse al lado mío y yo hacía seis cajas de tomate y él se hacía una, dos y después teníamos que sacar las cajas y yo las sacaba sola porque él no las podía levantar. Lo que pasa es que el no está acostumbrado a trabajar... se les dio todo, la escuela.

La madre agregó al respecto:

Tienen de todo. En cambio los cuatro más grandes, a la edad de los 10 iban a trabajar y este niño no. Para pedir es muy bueno. Todo el mundo en la escuela anda con celular y mirá mami: que tengo que ir a jugar a la pelota y así y todo lo que ve quiere. Y yo le digo que tiene que aprender a trabajar.

Por otro lado, el encargado de finca entrevistado al que hemos hecho mención previamente no habla de un conocimiento del trabajo sino de la responsabilidad:

...la gente joven que nunca ha hecho trabajos así te cuesta más; pero la gente grande ya no, es más consciente, más responsable ya. Por ahí cuando tomás gente nueva, que 
nunca ha hecho este trabajo, te cuesta mucho, porque no saben, les da lo mismo viste lo que está haciendo que lo que no, no le da mucha importancia.

Hay un reconocimiento del 'saber' pero vinculado a la implicancia y la 'responsabilidad', donde los saberes que no son considerados calificaciones se tornan en cualidades personales. Los saberes sobre el trabajo se ocultan detrás de diferentes cualidades personales que además se atribuyen a grupos determinados según el caso: mujeres, migrantes, norteños y los que aprendieron a trabajar desde pequeños.

En el relato citado se valora más la disposición a esforzarse en el trabajo que los conocimientos que permiten realizarlos de forma adecuada lo que se complementa con la iniciación temprana en el trabajo y el aprendizaje que ella conlleva ocultando el saber necesario para la realización de los trabajos al calificarlos como sencillos y fáciles de aprender. Esta simpleza emerge también en el relato de algunos trabajadores, como podemos en ver en el testimonio de Adriana, una trabajadora temporaria de 31 años residente en la zona rural circundante a la localidad de La Consulta, al preguntarle sobre cómo se aprenden los trabajos agrícolas:

Yo pienso que mirando y poniéndose a hacerlo vos aprendes así nomás. Así que yo sí, yo me animo a hacer todo trabajo que sea así en chacra. Yo no lo había hecho [se refiere a la cosecha de duraznos y manzanas]..., fui, lo hice y aprendí. Y me pagaban lo mismo que le pagaban a todos los demás.

Es habitual encontrar este tipo de descripciones del trabajo agrícola entre los propios trabajadores, pero no abundaremos en más testimonios de ese tipo ni en la multiplicidad de motivos sobre los que podrían fundamentarse dicha naturalización sobre la 'simpleza' o 'facilidad' de dichos trabajos. En cambio, trabajaremos sobre los testimonios de los trabajadores que expresan, aunque sea implícitamente, los saberes necesarios para trabajar y sobre las expresiones de los empresarios o encargados que describen los conocimientos necesarios para trabajar así como la importancia de dichos conocimientos para el desarrollo del proceso de trabajo y producción en su conjunto.

Por ejemplo Rosana, una trabajadora de 39 años, relata la forma en que se trabaja en la cosecha de cereza: 
Bueno, la cereza es un trabajo muy delicado, totalmente delicado. Es muy frágil, entonces hay que hacerlo con mucho cuidado. Se levanta toda con palo, lo que es como un ramito de uvas que trae tres o cuatro granos, ¿no es cierto? $\mathrm{O}$ sea que hay que levantarla con mucho cuidado para que salga con el palito.

En el caso de las cosechas que se realizan con escalera vemos que hay que clasificar las frutas para saber cuál cosechar, tomarla en forma adecuada para no romperla ni afectar la planta y sus futuros rendimientos y además manejar la escalera en forma adecuada para cuidar la seguridad del trabajador y tener la postura corporal que permite cosechar más rápido y mejor.

En el caso de la uva fina también se debe observar un cierto cuidado al cortarla, guardarla y para no romper la planta:

Por fichas...por cajas. Porque también depende de la uva...si es uva fina, va en cajitas, trabajamos muy cuidadosamente. Se levanta del parral muy delicadamente, se corta con tijera y se pone en la cajita con mucho cuidado y las van trasladando por el callejón...del callejón las llevan al depósito.

Otro trabajador, Raúl, relata las indicaciones previas que realizan los encargados antes de iniciar el proceso de trabajo haciendo visible la importancia de proceso de aprendizaje así como de los conocimientos previos:

El encargado se encarga de explicarle como se levanta la fruta y el contacto que usted puede tener con la fruta. Porque no es llegar y agarrarla a la fruta. Hay que observarla porque hay fruta que se levanta y hay fruta que no, porque todavía le falta. Entonces él le indica cómo la tiene que levantar a la fruta. Porque como ser la cereza, se levanta por partes...no se levanta toda junta. Primero se va levantando la más roja...la que ya está. Claro, y el encargado le explica cuándo ya está la fruta. Entonces, cuando ya está, la tiene que levantar, pero a lo mejor a la de al lado le falta un poquito y la tiene que dejar dos días más.

Las tareas de poda y raleo constituyen casos particulares pues hay distintas maneras de hacerlas que varían según la finca, tal como describe Luis, de 35 años, un trabajador temporario:

Es que año a año te van cambiando el tipo de poda [se refiere a la poda de vid]. Por ahí vas a un lado y te piden un tipo de poda, vas a otro lado y te piden otro tipo de poda, viste? Te cambia muchísimo. En casi todas las fincas... eso es algo que nunca lo terminás de aprender...nunca lo terminás de aprender, porque todos los días te están pidiendo algo distinto. Capaz que a lo mejor es una poda re estúpida, pero te la hacen cambiar, te la hacen cambiar. Igual que los raleos. Los raleos hay muchos que por 
ahí...por ejemplo por bandeja algunos te hacen dejar dos duraznos, otros te dejan quizás tres. Otros te hacen dejar cuatro, otros te hacen dejar uno. Porque depende cómo quieran, si quieren tamaño, si quieren fabriquero. Si es fabriquero, bueno, que sea chiquito no es nada porque maduran en fábrica, pero si es para exportación, tienen que ser grandes, así que tenés que dejar dos o tres como máximo por bandeja, porque cuanto más espacio le dejás, mas lugar le das al durazno para que crezca; porque si queda muy junto, cuando van creciendo se van chocando, entonces se van machucando. Y ese durazno ya no sirve. Para exportación no sirve porque se pudre en seguida.

En los testimonios de los trabajadores surgen muchas veces relatos de conflictos vinculados a los saberes requeridos para hacer los trabajos ya que en algunas ocasiones consideran que los 'encargados', los 'patrones' o los ‘ingenieros' están equivocados ya que su experiencia de trabajo contradice o no acuerda con las ordenes brindadas por estas figuras.

La disponibilidad de conocimientos es reconocida implícitamente pues su disposición es empleada, por ejemplo, para clasificar los trabajadores y distribuirlos en los puestos de trabajo que requieran y puedan aprovechar mejor dichos conocimientos. Veamos como lo narra un trabajador:

Lo que pasa es que a muchas mujeres por ahí las llevan... por ejemplo, en la cereza quedan para cajoneras, viste. Estar en los cajones, seleccionar la cereza, sacarla si está linda, si está fea. Ese trabajo es para las mujeres, generalmente, ...porque son las que tienen más paciencia para estar ahí. Aparte, siempre la mujer es más lerda que el hombre para trabajar. Entonces prefieren que el hombre esté cosechando y no que esté la mujer. Aparte, una escalera de 12 o 13 peldaños, la mujer no puede, en cambio el hombre sí. Esa es la diferencia. Y por ahí las plantas de cereza son muy altas y hay que subirse hasta la punta para poder sacar una cereza. Y las mujeres no suben. Las mujeres la mayoría andan por abajo. Muy pocas andan con escalera.

Por otro lado, la ausencia de definiciones acerca de los saberes requeridos para las tareas deja un apreciable margen de maniobra para las exigencias en torno al ritmo de trabajo y, por ende, a la explotación de la fuerza de trabajo, sobre todo en las tareas pagadas a destajo. Un cuadrillero y transportista de la zona relataba en esa línea los problemas con la forma en que los encargados gestionan y controlan el trabajo:

Bueno, eso es lo que le comentaba recién, no hay gente preparada para dirigirla a esa gente, a esos obreros. Porque hay algunos que se toman el látigo. Hay gente...grupos de gente de acá de la zona que no quiere trabajar por ejemplo en ciertas fincas [menciona en particular una empresa vitivinícola integrada de gran tamaño]. Porque dice que los encargados, los supervisores, como dice usted, exigen más de lo que en realidad una persona puede hacer. La experiencia que han tenido en otra empresa [menciona a otra empresa vitivinícola integrada de la zona] el año pasado fue justamente eso, le 
exigían...había un encargado que exigía que tenían que ser determinadas hileras de desbrote. Yo, por ejemplo, sé desbrotar, pero hay gente que no sabe desbrotar y ellos exigían de que rindieran hileras, entonces queda mal el trabajo. Ahora están pagando las consecuencias. Les cuento; la plantita cuando nace tiene...le dejan hoy día que vienen de vivero, nada más que la última yema. De la última yema nace el brotecito ya grande cuando se planta, por eso se está plantando Octubre, Noviembre, Diciembre, Enero y Febrero se ha plantado. Eso necesita...de las yemas nuevas nacen dos brotes, que es lo que uno debe saber que eso hay que sacarlo y dejar un solo tutor que vaya para arriba: despuntar y sacarle esos brotes. ¿Para qué? Para que se fortalezca. El año pasado como apuraban y todo lo demás, ¿qué ocurrió?, dejaron muchas doble yemas. Eso es muy tierno, hizo mucho bosque, pero se pasmó, se da cuenta...entonces ahora en este invierno, cuando fueron a podar se encontraron con que habían brotecitos así cortitos. Doble yema y todo lo demás, pero pasmada. Tuvieron que podar y perdieron...

A la clasificación de los trabajadores y su asignación a puestos de trabajo debemos agregar el proceso de selección de trabajadores, en cuyo fundamento encontramos tanto las cualidades personales como los conocimientos adquiridos ya sea en experiencias previas o en la actual. El mismo informante antes citado narra esta suerte de selección de personal:

...por el modo de trabajar diferente de las cooperativas y de los empresarios, desgraciadamente trabajan 90 días, los paran un mes y retoman. A la gente en realidad, como dice usted, que sabe trabajar, los retoman, ¿se da cuenta? Y el que no sabe trabajar...

La combinación de formas de contratación flexible, evaluación del rendimiento laboral y de cualidades personales (atributos de carácter) estructura el proceso de control y disciplinamiento de la fuerza de trabajo.

Un trabajador completa este panorama relatando lo que ocurre cuando un encargado considera que el trabajo no está bien hecho:

Siempre el encargado cuando uno por ahí se equivocó de fruta y la levantó...el encargado con mucha educación lo llama aparte de la cuadrilla y le dice 'Mirá, esto lo hiciste mal' o 'esto no va, no corresponde'. Le llama la atención una vez y le explica que dos veces no le puede llamar la atención porque si no el patrón va a tener problemas con él. ... Entonces bueno, si a la segunda vez, tercera vez, no le hizo caso y no respondió, al obrero lamentablemente lo suspenden porque no tiene capacidad para aprender.

Es vital para nuestro argumento señalar que el entrevistado designa un proceso de aprendizaje. Los conocimientos no sólo condicionan el proceso de trabajo sino que además, como ya hemos visto en testimonios previos, pueden condicionar, tanto en el caso de las 
tareas de cuidado y acondicionamiento de cultivos como en la misma cosecha, las potencialidades productivas futuras, como explicita esta trabajadora (María, 42 años):

No, le mira que coseche bien, que no corten los dardos, viste que vienen los dardos... si le cortás los dardos, el año que viene no te da fruta. ... Si, el tamaño de la fruta, todo. Si, por ahí te dan una hebillita, un alambrito así redondito, que tiene que entrar justo la manzana, la pera, viste?

Finalmente, es interesante agregar como contraste a la falta de 'certificación' o 'validación formal y reconocida' de estos saberes la valoración positiva de la posibilidad de tener estudios que hacen muchas trabajadoras/es. Tal el caso de Carmen (45 años):

Como está la situación, ahora, yo le digo a mi hijo que tenga un estudio. Si no tiene un estudio va a terminar de cualquier cosa.

En un mismo sentido, Erica, de 19 años, expresa:

Quisiera hacer una carrera y no trabajar al sol, para tener un buen futuro con mi hija y mi marido.

Vemos entonces que los conocimientos no son reconocidos como calificaciones laborales sino como aptitudes o atributos naturalizados a través de asociaciones de género o clase que ocultan su aprendizaje durante el proceso de trabajo tras el velo de disposiciones de carácter (responsabilidad, ganas de aprender, capacidad de esfuerzo), de analogías con otros roles sociales que explican la génesis de dichos atributos (fundamentalmente la maternidad) y de la asignación de atributos asociados a la masculinidad ya sea en sentido positivo, como la fuerza y la velocidad, o negativo como la falta de paciencia o atención.

\section{Naturalización del saber y descalificación del trabajo}

En este último apartado, completaremos el análisis mostrando cómo se gestiona y controla el proceso de trabajo, la forma en que se califica el desempeño de los trabajadores $\mathrm{y}$, finalmente, nos hemos preguntado cómo se valoriza en términos de remuneraciones y calificaciones las distintas tareas en relación a su importancia para el desarrollo del proceso de producción en su totalidad. Intentaremos mostrar de ese modo que en las descripciones 
del proceso de trabajo se asigna importancia y se mencionan saberes al describir las diferentes tareas pero que esos saberes no son valorados 'formalmente' ni reconocidos a través de mejores remuneraciones.

Una encargada de la gestión del proceso de una firma agroindustrial explica la importancia de la cosecha para la producción posterior y destaca de este modo la importancia de cosechar bien. Explica entonces que los productores que abastecen de duraznos a la empresa deben proveer la mayor proporción posible de duraznos aptos para industria, categoría en la que no entran los duraznos 'verdes', cosechados antes de tiempo. Para ello, la empresa, que contrata a los cuadrilleros que consiguen los cosecheros, paga sólo la fruta apta y monitorea la calidad de la fruta que se va cosechando:

\begin{abstract}
Nosotros vamos monitoreando, el va llevando un informe de lo que está pasando, entonces cuando nosotros vemos que están cosechando duraznos verdes ó pequeños por debajo de la medida, le avisamos porque lo consideramos inapto con valor cero para él. No se puede castigar al cosechador, pero si se puede castigar a un cuadrillero que está permitiendo que eso se haga; porque nosotros no podemos meter en la lata duraznos verdes. Te das cuenta, si ellos no lo dejan para una próxima pasada nos están cosechando duraznos que son pequeños y son verdes. Por ejemplo, cualquier fruta de carozo que te la saquen anticipadamente, te hacen perder una enorme cantidad de kilos porque después que se produce el primer cambio de color, la fruta crece el 3 y medio por ciento diario. Es decir que una fruta que la dejaste 5 días más, prácticamente tenes un $18 \%$ más de peso de fruta y hace que una fruta que te la mandaron a inapto que te la hace rechazar una máquina en línea, la puedas poner en primera, entonces nuestro planteo es claro: los contratamos para que cosechen y no para que hagan daño. Y si alguien está permitiendo que se haga daño tiene que pagar. Con ese planteo hemos logrado, la verdad, una muy buena conducta. ...Ahhhh, la selección por calidad la hacen las chicas. Cuando nosotros en el momento en el que llamamos, cuando la planilla nos está dando que tenemos un nivel muy alto de defectos en fruta; llamamos a equipos de mujeres que vienen a descartar. ... En esa tarea hay predominio femenino.
\end{abstract}

La descripción es suficientemente clara en relación a la importancia de hacer bien la tarea y a las consecuencias que hacerla mal puede tener para la producción y la rentabilidad del productor y de la agroindustria, así como a la adscripción a determinados puestos de trabajo por cualidades invisibilizadas como calificaciones laborales y atribuidas por motivos de género y clase.

Los cosecheros son trabajadores temporarios, tanto locales como migrantes, contactados por el cuadrillero, quien se encarga de la 'selección del personal', y contratados entonces por la empresa generalmente bajo la figura de permanente de temporada. Esto significa que cada temporada la empresa los vuelve a llamar obligatoriamente. La 
proporción de trabajadores permanentes es obviamente reducida en relación a la cantidad total de trabajadores.

Contratados los cosecheros, el cuadrillero se encarga de transmitir las instrucciones para la cosecha, y aquí nuevamente veremos emerger una serie de saberes que la empresa no desconoce pero que no tienen reconocimiento formal:

Dejamos que cada cuadrillero elija su recurso humano porque en definitiva los problemas de manejo de esa gente los va a sufrir el propio cuadrillero... Nosotros establecemos la calidad de la fruta que necesitamos, generalmente los cuadrilleros han sido ó productores u obreros que después compraron una camioneta y después empezaron a trasladar gente; pero como ellos conocen el oficio, muchas veces podes ver dentro de las quintas que le están enseñando hasta cómo parar la escalera para lograr rendimientos. Nosotros, después hemos mandando gente que son especialistas de tiempo y te marcan entre un modo de manejar la escalera y otro modo de manejar distinto la escalera, la diferencia de tiempo entre un cosechador y otro. ... No, no. La capacitación por el momento va por el cosechador. ... La idea, no hemos nosotros hecho la capacitación de cuadrilleros; solamente en normas de seguridad, en eso nosotros hemos tratado de trabajar, pero no del desarrollo del trabajo en sí.

Reconocer la fruta que debe ser cosechada y saber parar la escalera constituyen dos importantes 'saberes' que permiten una 'diferencia de tiempo'. Su enseñanza, en caso de que ello suceda, así como la selección de personal y el control del proceso de trabajo quedan en mano de los cuadrilleros, que reciben una paga adicional por tareas, responsabilidades y resultados. Si el trabajador conoce el oficio y realiza la tarea con velocidad y prestancia, entonces cosecha más y gana más. Cuando el cosechero realiza la tarea en forma apresurada pero cosecha fruta verde afecta la ganancia del cuadrillero y del productor y se expone a sanciones.

Precisamente, la persona entrevistada se refirió durante la entrevista al problema de la ‘implicación' en el trabajo por parte de los trabajadores cuando la tarea es al tanto.

La tarea al tanto siempre tratan de hacerlo lo más rápido posible, independientemente de que quede bien ó mal, y eso siempre es un problema que nosotros lo vemos en la gente joven, en la gente más joven. Al chico más joven no le interesa aprender a podar bien. A él le interesa sacar el día y tanto es así que en una de las encuestas que hicimos, al cosechero se le preguntaba: ¿cuánto usted considera que ha cumplido su objetivo? Y muchos nos contestaban: 'Cuando me puedo comprar el par de zapatillas que quiero'. Es decir, ustedes ven que él está completamente desconectado del objetivo fundamental de 'cuando entregué la cantidad de fruta que me propuse' ó 'cuando la fruta...' él está totalmente fuera del trabajo. 
Es decir, el objetivo enunciado por el trabajador lo deja, según la percepción de esta encargado de gestión de la empresa, 'fuera del trabajo' ya que su objetivo personal es valorado como contradictorio con el objetivo de la empresa. No ocurre lo mismo cuando el objetivo es realizado por quien sabe hacer las tareas y entonces asocia virtuosamente velocidad y habilidad, como expone el siguiente testimonio sobre la tarea de poda:

Porque poda, poda está generalizado en la zona como una actividad que aunque es considerada como muy importante no se consigue gente que quiera trabajar al día, porque tenés una gran diferencia entre el podador que sabe podar y el que es nuevo. La poda es una habilidad, entonces el buen podador siempre quiere podar al tanto. Entonces, lo que nosotros hacemos es tratar de captar siempre en nuestras cuadrillas los buenos podadores y empezamos las podas siempre temprano, para darle la posibilidad a estos podadores que están en nuestras cuadrillas de que terminen rápidamente. Para que te des una idea, el 30 de julio nosotros teníamos terminadas las podas y ellos terminan con nosotros y se van a trabajar a otros lados, hacen muchísimo dinero porque son los mejores.

En primer lugar se reconoce un saber que determina una habilidad y es importante para el proceso productivo. Pero casualmente ello ocurre porque la poda es una actividad considerada más crucial y también más difícil y, además, se realiza en un momento en el que no afluyen trabajadores migrantes en la misma cantidad que durante la cosecha. Sin embargo, la tarea también se realiza con trabajadores eventuales y se paga a destajo. La estrategia de la empresa para garantizarse los trabajadores en cantidad y calidad es realizar las podas 'temprano' para que luego los trabajadores puedan emplearse en otras fincas pero no capacitar un mayor número de trabajadores o pagar mejor la tarea.

Un aspecto interesante en relación a los conocimientos surge cuando la entrevistada, con quien la conversación fue tensa por momentos ya que entendía que juzgábamos negativamente su comportamiento y positivamente el de los trabajadores, intenta demostrar que no tiene prejuicios sobre los trabajadores. Consultada sobre los motivos de la contratación de mujeres para tareas de monitoreo de calidad la entrevistada contestó lo siguiente:

Yo, por qué la empresa lo decide no lo sé, supongo que por lo mismo que decide todo el mundo, por aquello de que está muy difundido en el medio que la mujer es mucho más observadora, porque clasificar significa tener la habilidad de mirar los detalles; yo te digo que en mi trabajo de finca tengo mujeres que no me han servido para calidad, por ejemplo. Tengo mujeres muy involucradas en los procesos de calidad de la cadena productiva, esposas... tengo un caso de una esposa de un encargado con un caso de 
escuela primaria terminada que yo te puedo decir que es mi mano derecha en la monitoreada de plagas y en la toma de decisiones en cuanto a calidad y destino de frutas, apoyando ella a su marido en el proceso, y tengo la esposa de otro que es maestra y que llegó un momento que contaba frutos y te puedo asegurar que no sabía para qué lo hacía. O sea, no se involucra en el proceso. Las habilidades, yo creo, que dependen de cuán involucrado esté en el proceso la persona a la que vos elegiste para realizar.

La comparación que establece la entrevistada se inicia con un intento de desnaturalizar la supuesta habilidad femenina para observar los detalles sosteniendo que ha habido trabajadoras que no lo han hecho bien. En la comparación se refuerza la idea de que una persona con un bajo nivel de educación formal, 'un caso de escuela primaria terminada', puede hacer mucho mejor el trabajo que una persona con un mayor nivel de educación formal, una 'maestra'. Pero ese rendimiento laboral no es finalmente remitido a un saber adquirido en el marco del aprendizaje de un proceso laboral sino a la capacidad de involucramiento en el proceso de trabajo, nuevamente una cualidad de carácter.

A preguntar si habitualmente era difícil conseguir trabajadores, la caracterización de los trabajadores se amplió, incorporando los planes de asistencia social otorgados por el Estado como una variable explicativa del poco apego al trabajo, señalada como la falta de una 'cultura del trabajo', e introduciendo la comparación con los trabajadores golondrinas:

Sí, sí. Nosotros por ejemplo, la gente nuestra ahora ya si no viene a ganar mucho..., inclusive más de una vez dicen: 'no, yo por esa plata no vengo a trabajar, tengo un plan. Mi mujer tiene un plan' [con tono despectivo] y el tema del plan se lo pasan por la nariz a uno cuando viene a ofrecer trabajo. Los mismos cuadrilleros se quejan de que los lugareños no quieren trabajar. El norteño [se refiere a los trabajadores golondrinas provenientes de las provincias del norte de Argentina como Tucumán, Salta, Jujuy y Santiago y también de Bolivia] viene al momento... ellos vienen a los trabajos al tanto, para los momentos que saben que van a ganar mucho dinero. Sabemos que son muy nómades, sabemos que al mismo tiempo cosechan la caña de azúcar, van al tomate y en Santiago, recién aparecen por acá en la zona y cuando empieza a hacer frío se van.

La comparación evidencia nuevamente un ocultamiento de realidades sociales detrás de una explicación aparentemente cultural. Los trabajadores migrantes de temporada, conocidos en la jerga como 'golondrinas', son generalmente trabajadores transitorios precarios que en sus lugares de origen se dedican a la agricultura y que también trabajan en actividades como la construcción y servicios, pero cuyos lugares de origen dependen intensamente de los ingresos generados en la temporada de cosechas de otras regiones en muchos casos coincidentes con los momentos de desempleo más agudo. Su nomadismo no 
es una preferencia cultural, su partida no se asocia a factores climáticos ni su llegada a una casualidad coincidente con la oportunidad de 'ganar mucho dinero'. En la continuidad de la investigación, hemos podido comprobar que los trabajadores migrantes (alrededor de unos cinco mil trabajadores sobre un total de veinte mil que se emplean exclusivamente en la zona del Valle de Uco) cubren períodos de desocupación y ganan lo mismo que los trabajadores locales representando este ingreso, en el caso de los trabajadores tucumanos, una suma similar a la que ganan en origen (Berger, Jimenez y Mingo, 2012).

La entrevistada interpretaba, como muchos argentinos, que los planes sociales tenían el efecto de hacer que las personas no quisieran trabajar y planteaba una mirada crítica a las posibilidades de discutir mejoras salariales producto del ingreso mínimo al que permitían acceder los mencionados planes:

\begin{abstract}
Me parece una barbaridad; si me dijeras mil becas para estudio, estoy de acuerdo; pero mil planes donde falta mano de obra es una vergüenza nacional. Te lo digo sinceramente. ...Yo te digo que estoy frente de una empresa donde me pasan más de una vez por la nariz y me dicen 'Yo, por ese dinero, me quedo a tomar mate porque por eso me pagan ciento cincuenta a mí y ciento cincuenta a mi mujer. Te lo digo en una empresa que los tiene en los libros, que les paga escolaridad, que tienen mutual, que tiene servicio médico, que tenemos servicio médico en las fincas todos los miércoles y aun así prefieren quedarse a tomar mate por 150, entonces por eso...
\end{abstract}

Hay que agregar que se trata de ocupaciones temporarias cuya extensión alcanza un promedio de tres o cuatro meses, con pago a destajo y jornadas no trabajadas y no pagas por lluvia y remuneraciones usualmente bajas. Cuando termina la cosecha, los empleados quedan en disponibilidad hasta que algunos de ellos vuelven a ser contratados para la poda; lo que en el caso de la empresa en la que trabaja la entrevistada representa un período de alrededor de dos meses:

Se termina la cosecha y el cuadrillero se va de la finca, desaparece y está terminando alrededor de entre el 3 y el 10 de marzo y nos volvemos a encontrar con ellos para la poda, eso es alrededor del 20 de mayo.

Habiendo comprendido la centralidad del rol de las 'monitoras de cosecha' que controlan la calidad de la fruta cosechada en relación al destino productivo que se le dará, preguntamos si se les pagaba un salario superior y la respuesta fue negativa, a pesar de señalar que las “descartadoras son un personaje fundamental en la cosecha porque maneja 
mucha información”. Las monitoras cumplen ese rol cuando se detectan problemas con la calidad de la cosecha pero si no es así son cosecheras y, según los informantes, allí se desempeñan mujeres porque dado que los hombres tienen más fuerza y eso les permite manejar mejor y más rápido la escalera, prefieren desempeñarse en 'tareas pagadas por tiempo'.

Por último, al preguntar a esta entrevistada cuáles eran las tareas mejores pagas, la respuesta fue que no hay tareas mejor pagas sino 'tareas en las que las personas ganan más porque ganan por producción'. Reformulamos la pregunta indagando sobre cuáles eran las tareas que a la empresa le interesaba que se hagan mejor y por lo tanto se pagaban un poco más, y obtuvimos esta respuesta:

Todas, todas. La empresa nunca paga un poco más, salvo en el caso del raleo, pagamos un poco más y necesitamos mucha gente.

Los testimonios apuntan a señalar la falta de reconocimiento social y salarial de los saberes necesarios, mencionados por trabajadores, encargados de finca y encargados del proceso de producción. Como hemos sostenido más arriba, ello se combina con formas de contratación flexible que agudizan la precariedad y vulnerabilidad de los trabajadores, les impide tener antigüedad, trabajo estable y un reconocimiento de sus calificaciones. Esto se observa en el relato de un ingeniero encargado del proceso de producción en una empresa importante de la zona dedicada a vitivinicultura y fruticultura:

\footnotetext{
En el área viñedos, aproximadamente estamos ocupando alrededor de 150 personas; en éste momento estamos trabajando con ése número, de los cuales 50 son efectivos y el resto es personal temporario. De éstos temporarios, estoy hablando de gente que hace 6 ó 7 años que trabaja pero bueno, sigue en la categoría de temporarios. ... Exactamente, por temporada, creo que cada tres...no, eso no lo tengo muy claro, pero creo que cada tres meses se los para un tiempo. Se los para quince días. Lo que tenemos normalmente, tenemos una...otra categorización los permanentes que normalmente son los capataces, los tractoristas, los regadores y hay aparte obreros, peón común de campo que son también los que llevan más años, pero esos mismos peones comunes hacen el mismo trabajo que los temporarios. ... (fichero y recorredor) salen de los permanentes.
}

Es decir, según este testimonio, la mayoría de los obreros son peones de campo salvo los capataces, los tractoristas y los regadores, unas categorías en las que se reconoce un conocimiento asociado a un puesto de trabajo. Pero en el caso de los trabajadores que no 
revistan dentro de esas categorías son peones generales, trabajadores sin un saber ni tareas específicas reconocidos formalmente.

\section{Reflexiones finales}

En este artículo hemos tratado de comprender el proceso por el cual el trabajo agrícola es desvalorizado. Entendemos que dicha desvalorización es producto de la falta de reconocimiento social y formal de los saberes involucrados en la realización de las tareas agrícolas a las que podemos agregar también trabajos realizados en la agroindustria.

A partir del trabajo de campo y de nuestras indagaciones (Mingo y Berger: 2009; Mingo, 2012) interpretamos que dicha desvalorización se fundamenta en un proceso de social que reproduce en forma constante sus condiciones de posibilidad. En este artículo hemos intentado desandar ese proceso que entendemos tiene un punto de apoyo en las condiciones de reproducción de los hogares de los trabajadores (Berger y Mingo, 2011) que impulsan la incorporación al trabajo entre los diez y quince años y activan de ese modo una tensión con la continuidad de trayectorias educativas, ya de por sí realizadas en condiciones desventajosas en comparación con otros sectores de la población.

Pero esa incorporación, además de abrir el camino para la naturalización de condiciones de trabajo y de vida y constituir la génesis de procesos de individuación articulados alrededor de la figura de trabajador independiente que desplaza y desalienta la figura del estudiante, también expresa uno de los fundamentos prácticos del ocultamiento de los saberes necesarios para desempeñarse como trabajador agrícola.

Por consiguiente, lo que 'califica' a un trabajador no son sus conocimientos y capacidades certificadas por su trayectoria educativa y laboral, que generalmente es desconocida, sino aquellas cualidades personales que son observadas durante su desempeño como trabajador. Lo que discursivamente se califica en primer lugar es su comportamiento, expresado en atributos como responsabilidad, esfuerzo y constancia.

Por otro lado, la presunta 'sencillez' de los trabajos está asociada a población con escaza formación, relegada socialmente, donde se supone que la cultura del trabajo se ve afectada por la asistencia del Estado (ejemplificada en los planes sociales) y cuyas ambiciones no siempre están en línea con las prácticas y expectativas que expresan el deseo de progreso socioeconómico. 
Como contrapartida, el aprendizaje de los trabajos no es reconocido formalmente y, nada casual, las trayectorias educativas no son asociadas con la continuidad como trabajadores agrícolas sino con la posibilidad de desempeñarse en otros sectores y trabajos percibidos como 'buenos trabajos'.

Complementariamente, los conocimientos asociados al trabajo agrícola son invisibilizados detrás de cualidades personales asociadas a la conducta pero no al aprendizaje. Así, los testimonios de los entrevistados apuntan a que un buen trabajo no es un trabajo en la agricultura y que para tener un buen trabajo hay que estudiar.

Es decir, interpretamos, que un buen trabajo es 'otro trabajo' y requiere de otra forma de aprendizaje, diferente de aquella casi inmediata, o apenas mediada por vínculos familiares, vecinales o amicales, vínculos de aprendizaje informal cuyo resultado no es reconocido como calificación ya que esos saberes no se hallan certificados. Otro aprendizaje, otro trabajo, otra vida posible, inaccesible para quien sea trabajador agrícola.

\section{Referencias}

BERGER, Matías; BOBER, Gabriel; FABIO, Francisco; MINGO, Elena; y NEIMAN, Melina. “Legalizar la precariedad?: La forma cooperativa en la movilización de mano de obra en el agro”. En: CROSS, Cecilia y BERGER, Matías (comp.). La producción del trabajo asociativo: condiciones, experiencias y prácticas en la economía social. Buenos Aires: CICCUS, 2010.

BERGER, Matías y MINGO, Elena. "Condiciones de reproducción e inserciones laborales de los trabajadores agrícolas en el Valle de Uco, provincia de Mendoza". En: Revista Theomai, n.24. Buenos Aires: Red de Estudios sobre Sociedad, Naturaleza y Desarrollo, 2011.

CERDÁ, Juan Manuel: "El mercado de trabajo y las condiciones de vida en Mendoza a comienzos del siglo XX”. En: Actas de las IV Jornadas Interdisciplinarias de Estudios Agrarios y Agroindustriales. Buenos Aires: Facultad de Ciencias Económicas, 2005.

CERDÁ, Juan Manuel: "Mercado de trabajo y condiciones de vida en Mendoza a comienzos del siglo XX”. En: Revista Mundo Agrario, n.12. Buenos Aires: Centro de Estudios Histórico-Rurales, 2006.

GOMÉZ, Daniel: “La vitivinicultura argentina: génesis, consolidación y crisis de un proceso de industrialización con base agraria”. En: Actas de las II Jornadas Interdisciplinarias de Estudios Agrarios y Agroindustriales, noviembre de 2001. Buenos Aires: Facultad de Ciencias Económicas, 2001.

MATEU, Ana María: La Vitivinicultura mendocina entre 1870 y 1920: la génesis de un modelo centenario. El vino y sus revoluciones. Una antología histórica sobre el desarrollo 
de la industria vitivinícola argentina. Mendoza: Editorial de la Universidad Nacional de Cuyo, 2007.

MINGO, Elena y BERGER, Matías: "Asalariados rurales en el Valle de Uco (provincia de Mendoza)". En: Revista Mundo Agrario, v.10, n.19. Buenos Aires: Centro de Estudios Histórico-Rurales, 2009.

MINGO, Elena. El trabajo de las mujeres en la agricultura y la agro-industria del Valle de Uco, provincia de Mendoza. Trabajo asalariado, trabajo doméstico y división sexual del trabajo. (Tesis Doctoral). Buenos Aires: Facultad de Ciencias Sociales de la Universidad de Buenos Aires, 2012.

OSPITAL, María Silvia. "Modernización estatal y regulación económica en provincias vitivinicultoras. Mendoza 1936-1946". En: Revista Mundo Agrario, v. 9 n. 18. Buenos Aires: Centro de Estudios Histórico-Rurales, 2009.

RICHARD-JORBA, Rodolfo. "Transformaciones económicas y formación del mercado de trabajo libre en Mendoza. El trabajo rural entre la segunda mitad del siglo XIX y los albores del XX". En: Actas del 5 Congreso Nacional de Estudios del Trabajo, 2001. Buenos Aires: Asociación Argentina de Especialistas en Estudios del Trabajo, 2001.

RICHARD-JORBA, Rodolfo. "Inmigración, mercado de trabajo y vitivinicultura. Mendoza y San Juan 1869-1914". En: Actas de las IV Jornadas Interdisciplinarias de Estudios Agrarios y Agroindustriales, 2005. Buenos Aires: Facultad de Ciencias Económicas, 2005.

Recebido em: 06/04/2012

Aprovado em: 25/05/2012 